\title{
Killing Oral Bacteria Using Metal-Organic Frameworks
}

Piao Cao ${ }^{a, 1}$, Xiaojun Wu ${ }^{b, 1}$, Wenbo Zhang ${ }^{a}$, Ling Zhao ${ }^{a, c}$, Weizhen Sun ${ }^{a, *}$, and Zisheng Tang ${ }^{d, e, f, *}$

a State Key Laboratory of Chemical Engineering, East China University of Science and Technology, Shanghai 200237, China

${ }^{b}$ Department of Neurosurgery, Renji Hospital, School of Medicine, Shanghai Jiao Tong University, 1630 Dongfang Road, Shanghai 200127, China

c School of Chemistry \& Chemical Engineering, XinJiang University, Urumqi 830046, China

d Department of Endodontics, Shanghai Ninth People's Hospital, College of Stomatology, Shanghai Jiao Tong University School of Medicine, Shanghai 200011, China

e National Clinical Research Center of Oral Diseases, Shanghai 200011, China

${ }^{f}$ Shanghai Key Laboratory of Stomatology \& Shanghai Research Institute of Stomatology, Shanghai 200011, China

${ }^{*}$ Corresponding author.

E-mail address: sunwz@ecust.edu.cn (W. Sun). Tangzisheng163@163.com (Z. Tang).

${ }^{1}$ These authors contributed equally to this work. 


\section{Synthesis details of MOFs}

The synthesis of p-MOF. ${ }^{1} 150 \mathrm{~mL}$ deionized water was added to a $250 \mathrm{~mL}$ round-bottomed flask and heated to $80{ }^{\circ} \mathrm{C}$. Then $0.7473 \mathrm{~g}$ p-cyanobenzoic acid and $0.5144 \mathrm{~g}$ trimethylamine were weighed and added to the flask, respectively. $30 \mathrm{~mL}$ silver nitrate solution with molar ratio of 1:1:1 for p-cyanobenzoic acid, trimethylamine and silver nitrate was slowly added to the flask with continuous stirring. After $2 \mathrm{~h}$ reaction, the unreacted materials in the solution were removed using suction filter and the obtained solution was placed in the dark for 3 weeks to yield white needle crystal precipitation. The white powder solid, namely $[\mathrm{AgL}]_{\mathrm{n}} \bullet \mathrm{nH}_{2} \mathrm{O}(\mathrm{p}-\mathrm{MOF})$, was obtained after separation and vacuum drying.

The synthesis of MOF-5. ${ }^{2} 1.6613 \mathrm{~g}$ terephthalic acid (PTA) and $5.9498 \mathrm{~g} \mathrm{Zn}\left(\mathrm{NO}_{3}\right)_{2} \bullet \mathrm{nH}_{2} \mathrm{O}$ were added to a $250 \mathrm{~mL}$ beaker, then $100 \mathrm{~mL} \mathrm{N,N-dimethyl} \mathrm{formamide} \mathrm{(DMF)} \mathrm{was} \mathrm{added} \mathrm{to} \mathrm{fully}$ dissolve. $10 \mathrm{~mL}$ trimethylamine (TEA) was introduced into the beaker under continuous electromagnetic agitation. After $2 \mathrm{~h}$ reaction at room temperature, the liquid was removed by centrifugal filtration and the obtained product was immersed in $30 \mathrm{~mL}$ DMF for $24 \mathrm{~h}$ and then centrifuged for separation. The above steps were repeated for three times. Finally, the product was dried overnight in a vacuum oven at $60^{\circ} \mathrm{C}$ to give a white powdery solid, namely MOF-5.

The synthesis of ZIF-8. ${ }^{3} 3.2812 \mathrm{~g}$ 2-methylimidazole and $2.9725 \mathrm{~g} \mathrm{Zn}\left(\mathrm{NO}_{3}\right) \bullet 6 \mathrm{H}_{2} \mathrm{O}$ was dissolved in $75 \mathrm{~mL}$ deionized water and $50 \mathrm{~mL}$ methanol in a $250 \mathrm{~mL}$ beaker. After $2 \mathrm{~h}$ reaction time at room temperature, the upper liquid was removed and the obtained polyhedral crystal product was collected and washed in $30 \mathrm{~mL}$ DMF for three times. Finally, the product was dried overnight in a vacuum oven at $60{ }^{\circ} \mathrm{C}$ to give a white powdery solid, namely ZIF-8.

\section{PXRD of synthesized MOFs}


p-MOF, MOF-5 and ZIF-8 were synthesized using the solvothermal method. The powder X-ray diffraction (PXRD) patterns of the synthesized MOFs in this work were shown Figure S1. The experimental patterns match well with the corresponding simulated patterns of p-MOF, MOF-5 and ZIF-8, demonstrating that the products are pure-phase MOF crystals and the target products.
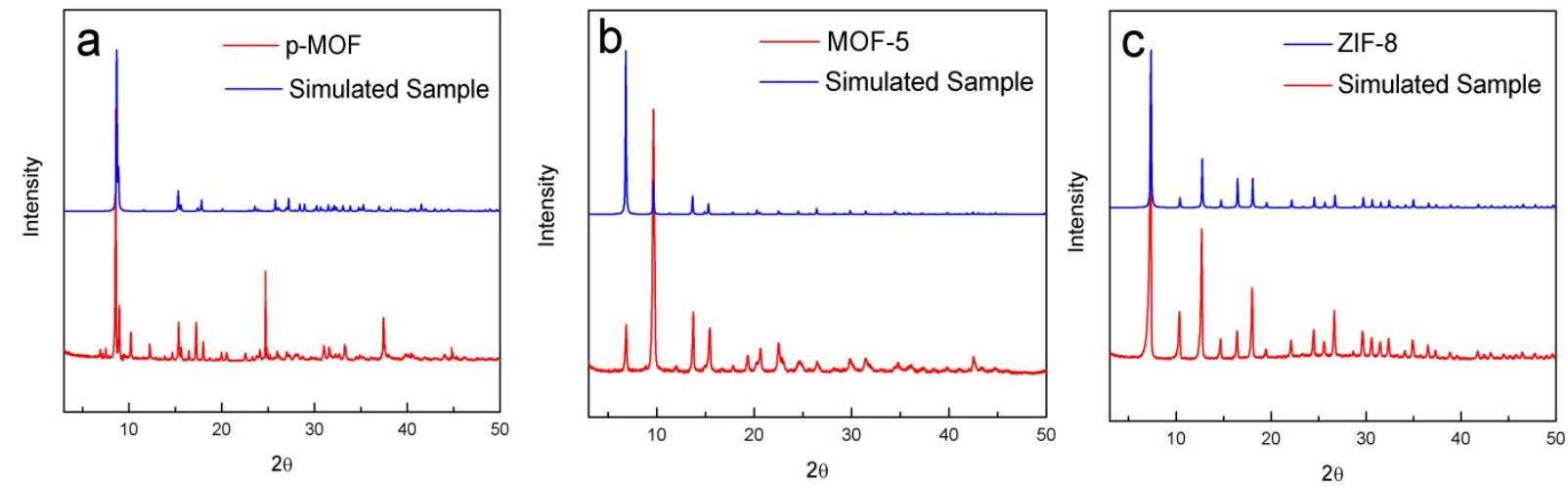

Figure S1. PXRD patterns of p-MOF (a), MOF-5 (b), ZIF-8 (c).

\section{FT-IR of synthesized MOFs}

The results of FT-IR spectroscopy are shown in Figure S2. The characteristic peak in experimental spectrogram matches well with the corresponding spectrogram from references ${ }^{1,4-5}$.
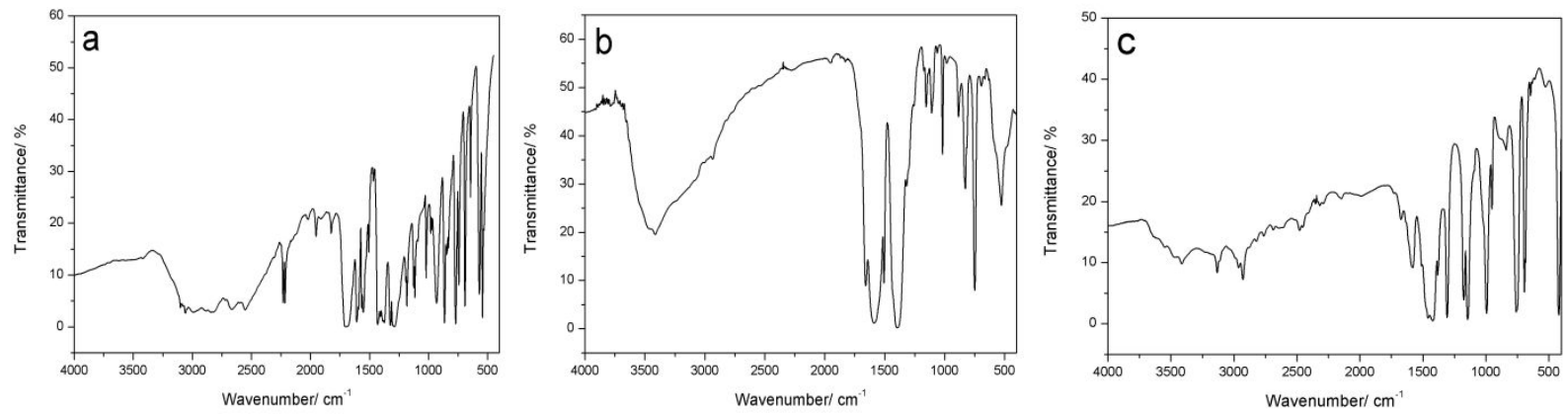

Figure S2. FT-IR spectroscopy of p-MOF (a), MOF-5 (b), ZIF-8 (c).

\section{TGA traces of synthesized MOFs}

To investigate the thermal stability of synthesized MOFs materials in this work, the thermal gravimetric analysis (TGA) were performed under an $\mathrm{N}_{2}$ atmosphere. The TGA results of p-MOF are 
shown in Figure S3(a). The first weight loss step is related to desorption of guest molecules. Another mass change of around $45 \%$ occurs at about $270{ }^{\circ} \mathrm{C}$, which is in good agreement with the reference ${ }^{1}$, ${ }^{6}$. Figure S3(b) indicates that MOF-5 material maitain thermal stability below $400{ }^{\circ} \mathrm{C}$. There is only one main weight loss around $450{ }^{\circ} \mathrm{C}$, meaning that guest molecules are evacuated completely. For ZIF-8 in Figure S3(c), the gradual weight loss is ascribed to the removal of guest molecules and unreacted species. The results reveal that ZIF-8 material synthesized in this work is thermal stable up to $400{ }^{\circ} \mathrm{C}$.
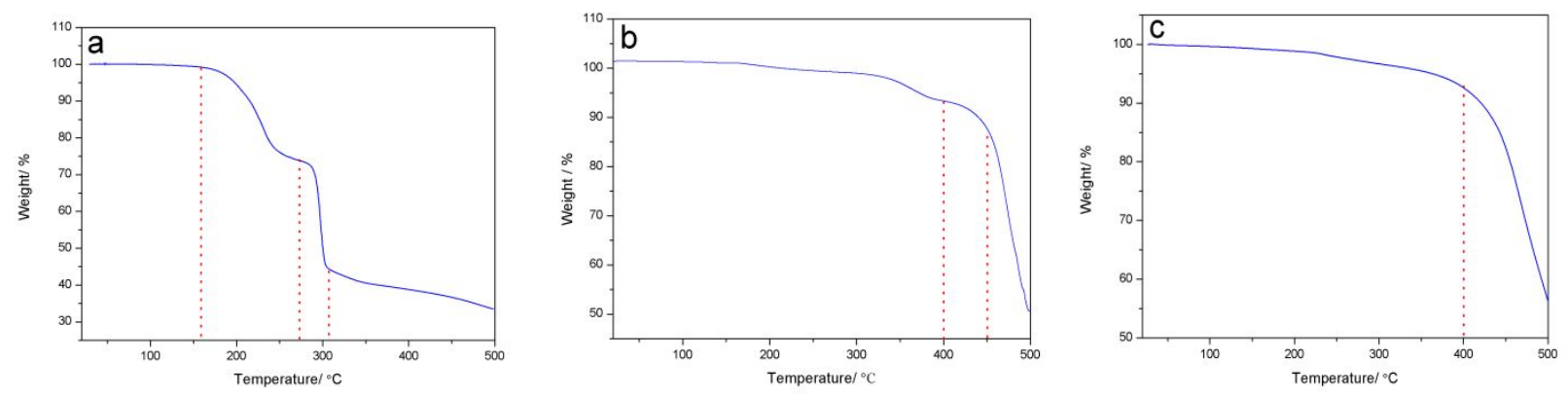

Figure S3. TGA traces of of p-MOF (a), MOF-5 (b), ZIF-8 (c).

\section{REFERENCE}

(1) Wang, M.-S.; Guo, S.-P.; Li, Y.; Cai, L.-Z.; Zou, J.-P.; Xu, G.; Zhou, W.-W.; Zheng, F.-K.; Guo, G.-C. A direct white-light-emitting metal- organic framework with tunable yellow-to-white photoluminescence by variation of excitation light. Journal of the American Chemical Society 2009, 131 (38), 13572-13573.

(2) Tranchemontagne, D. J.; Hunt, J. R.; Yaghi, O. M. Room temperature synthesis of metal-organic frameworks: MOF-5, MOF-74, MOF-177, MOF-199, and IRMOF-0. Tetrahedron 2008, 64 (36), 8553-8557.

(3) Park, K. S.; Ni, Z.; Côté, A. P.; Choi, J. Y.; Huang, R.; Uribe-Romo, F. J.; Chae, H. K.; O'Keeffe, M.; Yaghi, O. M. Exceptional chemical and thermal stability of zeolitic imidazolate frameworks. Proceedings of the National Academy of Sciences 2006, 103 (27), 10186-10191.

(4) Qian, K.; Fang, G.; Wang, S. A novel core-shell molecularly imprinted polymer based on metalorganic frameworks as a matrix. Chemical Communications 2011, 47 (36), 10118-10120.

(5) Sun, C.-Y.; Qin, C.; Wang, X.-L.; Yang, G.-S.; Shao, K.-Z.; Lan, Y.-Q.; Su, Z.-M.; Huang, P.; Wang, C.-G.; Wang, E.-B. Zeolitic imidazolate framework-8 as efficient pH-sensitive drug delivery vehicle. Dalton Transactions 2012, 41 (23), 6906-6909.

(6) Aghajanloo, M.; Rashidi, A. M.; Moosavian, M. A. Synthesis of zinc-organic frameworks nano adsorbent and their application for methane adsorption. Journal of Chemical Engineering \& Process 
Technology 2014, 5 (5), 1. 